\title{
An optimal fuel-injection policy for performance enhancement in internal combustion engines
}

\author{
V H GUPTA ${ }^{\prime}$ and SHALABH BHATNAGAR $* . \dagger .2$ \\ 1 9, Anand Nagar, Raipur 492001, India \\ ${ }^{2}$ Department of Electrical Engineering, Indian Institute of Science, \\ Bangalore 560012, India \\ † Present address: Institute of Systems Research, 2269 A V Williams Building, \\ University of Maryland, College Park, Maryland, MD 20742, USA \\ e-mail: shalabh@isr.umd.edu
}

\begin{abstract}
A fuel-injection internal combustion engine system is considered, wherein supply of fuel to the engine is controlled with the twin purposes of maximizing power output and minimizing fuel wastage. The system is modelled as a controlled Markov chain and a feed-back optimal control policy is obtained for the long-run average reward optimality criterion using Markov decision theory.
\end{abstract}

Keywords. Dynamic programming/optimal control; infinite state Markov chain; long run average reward; optimal policy; fuel injection control in IC engines.

\section{Introduction}

Internal combustion (IC) engines are widely used in a variety of applications and almost most importantly in automobiles. A large number of such machines use the two-stroke petrol engines. One of the gravest problems with two-stroke petrol engines is the unreliable combustion, which leads to cycles (a cycle comprises one revolution of the crank and is equivalent to two strokes of the piston) that do not produce any power and also release unignited fuel which goes to the exhaust and gets wasted. Twenty-five percent of the cycles being 'powerless' is quite common even in the latest engines. Until recently, fuel was supplied to the system using a fuel-air mixer (technically known as carburettor) almost in all cases. This carburettor being in line with the air-flow of the system would supply the fuel-air mixture continuously to the combustion chamber system. However, in recent times, there is a shift to 'direct petrol injection' systems, where air supply is done independently and the fuel is directly injected into the system, once every cycle. This has rendered the

*Author for correspondence 
system amenable to fuel injection control, since one could decide whether the fuel was to be injected or not. We refer the reader to Moskwa \& Hedrics (1992) and Jones et al (1995), and references therein, for the latest trends in this direction.

In the following, we propose a stochastic model of the system, and also propose strategies for control. The key idea is to predict on-line, the occurrences of 'powerless' cycles stochastically, and to cut fuel at those random instants, thus minimizing fuel wastage without compromising on the power output of the engine.

\section{The problem}

We consider an engine with fuel injection operating in a steady state. The two binary variables that are of interest to us every $i$ th cycle are $x_{i}$ and $y_{i}$, which are defined as follows. $x_{i}=0$, if fuel is not injected, and $x_{i}=1$, if fuel is injected. Similarly, $y_{i}=0$, if combustion does not take place, and $y_{i}=1$, if combustion does take place. We note that if we define $z_{i}=x_{i}-y_{i}$, then $z_{i}$ is also a binary variable such that $z_{i}=0$, if $x_{i}=y_{i}=1$ or $x_{i}=y_{i}=0$, and $z_{i}=1$, if $x_{i}=1$ and $y_{i}=0$. It is evident that the possibility $x_{i}=0$ and $y_{i}=1$ is impossible, since there can be no combustion without any fuel being injected. Clearly, if $z_{i}=0$, then there is no wastage of fuel, and if $z_{i}=1$, then fuel in that cycle is wasted. Hence, $\sum_{i} z_{i}$ is a 'measure' of the total fuel wastage. Also note that $\sum_{i} y_{i}$ is a 'measure' of the total power output. If we further denote by non-negative constants $B$ and $A$, the cost of fuel per cycle and the profit or gain from power output per cycle respectively, then the problem reduces to the combined task of maximizing $\sum_{i} A y_{i}$ and minimizing $\sum_{i} B z_{i}$. Accordingly, we choose a cumulative reward function $C$, defined by $C=A \sum_{i} y_{i}-B \sum_{i} z_{i}$. Here, we also note that $x_{i}$ and $y_{i}$ being random variables, we cannot directly maximize $C$, though we could maximize the expectation of $C$. Noting that $y_{i}$ are random variables, we also make the following assumptions.

Assumptions A. (1) $\left\{y_{i}\right\}$ is such that whenever $y_{i}=0$, (2) and (3) below (of (A)) hold from $i$ onward and this does not depend on the value of $i$.

(2) We denote by $p_{1}, p_{2}, \ldots$ the probabilities $\operatorname{Prob}\left\{y_{i+1}=1 \mid y_{i}=0, x_{i+1}=1\right\}=p_{1}$, and for $k>1$,

$$
\operatorname{Prob}\left\{y_{i+k}=1 \mid y_{i+k-1}=1, \ldots, y_{i+1}=1, y_{i}=0, x_{i+k}=1\right\}=p_{k},
$$

where the vertical bar denotes conditional probability.

(3) $1>p_{1} \geq p_{2} \geq p_{3} \geq \cdots$ etc.

We now explain the physical significance of $A(1)-A(3)$.

$A(1)$ implies that whenever there is a 'powerless' cycle, the whole system begins afresh and what happened before such a cycle does not affect what happens afterwards. This is quite reasonable, since otherwise one will have to assume either an infinite memory system or an arbitrarily assigned finite memory system.

$\mathrm{A}(2)$ means that combustion history probabilistically determines its future. One should note here that this structure must be corroborated by 'experimental observation'.

$\mathrm{A}(3)$ means that as the sequence of fuel utilization (fuel-non-wastage) becomes longer, the chances of encountering fuel wastage increase. This is possibly the outcome of the 
pessimism that the longer things go 'right', the chances that they go 'wrong' increase! The restriction $p_{1}<1$ is mild and merely allows for consecutive 'powerless' cycles. However $p_{1}$ can be as close to 1 as we want.

In what follows, we shall formulate this problem as a controlled Markov chain and apply Markov decision theory to obtain the optimal closed loop feedback control policy.

We shall now describe the manner in which this controlled Markov chain is constructed, in lieu of (A). We use $\{0,1\}$ as the control set with $x_{i}$ as the control at the $i$ th step. Hence, at any stage we pick $x_{i}=1$ as the control if we decide to send fuel and choose $x_{i}=0$ otherwise.

Let $\left\{X_{n}\right\}$ represent the controlled Markov chain on the state space of nonnegative integers with $\{0,1\}$ above as the control set. Let $X_{0}=j$ represent the initial state, for some integer $j$. Then for any integer $i \geq 1, X_{i}=j+i$ if $y_{1}=y_{2}=\cdots=y_{i}=1$, else if $y_{k}=0$ for some integer $k, X_{k}=0$ as well. Hence for $i<n$, if $X_{n}=i$, then $X_{n-i}=0$. Therefore any state $i$ in the state space of the Markov chain corresponds to the $i$ th consecutive 1 in the $y_{n}$ sequence. The moment $y_{n}=0$ for some $n$, the Markov chain jumps back to state 0 . Let $p(i, j, a)$ represent the transition probability of moving from state $i$ to state $j$ when action or control $a \in\{0,1\}$ is chosen. The transition probabilities then are:

$$
p(i, i+1,1)=p_{i+1}, \quad p(i, 0,1)=1-p_{i+1}, \quad \forall i \geq 0,
$$

and $p(i, 0,0)=1, \forall i \geq 0$. Here $p_{i}$ are as in (A).

Let $\hat{R}(i, a)$ represent the one-step reward random variables when the Markov chain is in state $i$ and action $a$ is chosen. In lieu of the cumulative reward function $C$ defined earlier, we define $\hat{R}(i, a)$ as follows:

$$
\begin{aligned}
\hat{R}(i, 1) & =A \quad \text { w.p. } p_{i+1}, \\
& =-B \quad \text { w.p. } 1-p_{i+1}
\end{aligned}
$$

$\forall i \geq 0$ and $\hat{R}(i, 0)=0$ w.p. $1, \forall i \geq 0$. Here and everywhere else w.p. stands for "with probability'. Now let $R(i, a)$ be the one-step expected reward in state $i$ when action $a$ is chosen. Then,

$$
R(i, 1)=A \cdot p_{i+1}-B\left(1-p_{i+1}\right)=(A+B) \cdot p_{i+1}-B, \forall i \geq 0,
$$

and $R(i, 0)=0, \forall i \geq 0$.

A policy is defined as a rule for selecting actions and can in general be randomized. Let for any policy $\pi$ and initial state $i$,

$$
\phi_{\pi}(i)=\liminf _{n \rightarrow \infty} \frac{E_{\pi}\left[\sum_{j=0}^{n} R\left(X_{j}, a_{j}\right) / X_{0}=i\right]}{n+1} .
$$

Here $E_{\pi}$ represents the expected value under policy $\pi, \phi_{\pi}(i)$ represents the average expected return per unit time when policy $\pi$ is employed and initial state is $i$. We shall say that a policy $\pi^{*}$ is average reward optimal if $\phi_{\pi^{*}}(i)=\max _{\pi} \phi_{\pi}(i), \forall i$. Our objective shall be to find $\pi^{*}$ from the class of all policies. 


\section{The optimal policy}

Let $V_{\alpha}$ represent the value function for the $\alpha$-discounted reward criterion defined as:

$$
V_{\alpha}(i)=\max _{\pi} E_{\pi}\left[\sum_{n=0}^{\infty} \alpha^{n} R\left(X_{n}, a_{n}\right) / X_{0}=i\right],
$$

where the discount factor $\alpha \in(0,1)$. The dynamic programming equation for the $\alpha$ discounted reward tells us (Ross 1983, p. 31)

$$
V_{\alpha}(i)=\max _{a}\left\{R(i, a)+\alpha \sum_{j} p(i, j, a) V_{\alpha}(j)\right\} .
$$

Since we have only two controls 0 and 1 , the above equation in our case becomes

$$
V_{\alpha}(i)=\max \left\{R(i, 1)+\alpha p_{i+1} V_{\alpha}(i+1)+\alpha\left(1-p_{i+1}\right) V_{\alpha}(0), \alpha V_{\alpha}(0)\right\},
$$

for $i \geq 0$. In what follows, we shall first show that $V_{\alpha}(i)$ is nonincreasing in $i$.

Lemma 1. Under $(A), R(i, 1)$ is nonincreasing in $i$.

Proof. $R(i+1,1)=(A+B) \cdot p_{i+2}-B \leq(A+B) \cdot p_{i+1}-B=R(i, 1)$, the inequality following because of $\mathrm{A}(3)$. This is true for every $i$. The claim follows.

\section{PROPOSITION 1}

Under $(A), V_{\alpha}(i)$ is nonincreasing in $i$.

Proof. Consider the value iteration form of (3) (see for instance, chapter II.3 of Ross 1983 for value iteration). We have

$$
\begin{aligned}
V_{\alpha}^{n}(i)=\max & \left\{R(i, 1)+\alpha p_{i+1} V_{\alpha}^{n-1}(i+1)\right. \\
& \left.+\alpha\left(1-p_{i+1}\right) V_{\alpha}^{n-1}(0), \alpha V_{\alpha}^{n-1}(0)\right\},
\end{aligned}
$$

$\forall i \geq 0, n \geq 1$. We shall first show by induction that $V_{\alpha}^{n}(i)$ is nonincreasing in $i$ for every $n$, and then we shall show that $V_{\alpha}^{n}(i) \rightarrow V_{\alpha}(i)$ as $n \rightarrow \infty$, thereby implying the claim.

Let $V_{\alpha}^{1}(i)=R(i, 1)$. Then, by the previous lemma, $V_{\alpha}^{1}(i+1) \leq V_{\alpha}^{1}(i)$. Now assume that $V_{\alpha}^{n-1}(j)$ decreases in $j$. So, as in (4), we have for $i+1$,

$$
\begin{aligned}
V_{\alpha}^{n}(i+1)=\max & \left\{R(i+1,1)+\alpha p_{i+2} V_{\alpha}^{n-1}(i+2)\right. \\
& \left.+\alpha\left(1-p_{i+2}\right) V_{\alpha}^{n-1}(0), \alpha V_{\alpha}^{n-1}(0)\right\} .
\end{aligned}
$$

We need to consider only the first terms in the arguments on the RHS of (4) and (5), since the second terms are identical. We have,

$$
\begin{aligned}
& R(i, 1)+\alpha p_{i+1} V_{\alpha}^{n-1}(i+1)+\alpha\left(1-p_{i+1}\right) V_{\alpha}^{n-1}(0) \\
& \quad=R(i, 1)+\alpha p_{i+1}\left(V_{\alpha}^{n-1}(i+1)-V_{\alpha}^{n-1}(0)\right)+\alpha V_{\alpha}^{n-1}(0) .
\end{aligned}
$$

Now since $V_{\alpha}^{n-1}(j)$ is nonincreasing in $j$, we have

$$
V_{\alpha}^{n-1}(i+2) \leq V_{\alpha}^{n-1}(i+1) \leq \cdots \leq V_{\alpha}^{n-1}(0),
$$


so, $V_{\alpha}^{n-1}(i+1)-V_{\alpha}^{n-1}(0) \geq V_{\alpha}^{n-1}(i+2)-V_{\alpha}^{n-1}(0)$. Now, by lemma 1 above, we have the LHS of $(6) \geq R(i+1,1)+\alpha p_{i+2}\left(V_{\alpha}^{n-1}(i+2)-V_{\alpha}^{n-1}(0)\right)+\alpha V_{\alpha}^{n-1}(0)$ or, $V_{\alpha}^{n}(i) \geq$ $V_{\alpha}^{n}(i+1), \forall i \geq 0$. Hence, $V_{\alpha}^{n}(i)$ is nonincreasing in $i, \forall n$ by induction. Since $R(i, 0)=0$, $\forall i$, it can be easily seen by (2) and lemma 1 that $\left|V_{\alpha}(i)\right| \leq|R(0,1)| /(1-\alpha)$. Now as in Ross $\left(1983\right.$, p. 36) $V_{\alpha}(i)=\lim _{n} V_{\alpha}^{n}(i)$, uniformly in $i$. Hence $V_{\alpha}(i)$ is nonincreasing in $i$.

Let $T=\min \left\{n>0 \mid X_{n}=0\right\}$ represent the first time the Markov chain $\left\{X_{n}\right\}$ hits state 0 starting from any state. Note that $T$ is a random time. Let $f_{\alpha}$ be the $\alpha$-discount optimal policy. Further, let $f$ be the policy that chooses action 1 for all $n$. Now clearly, $E_{f_{\alpha}}\left[T / X_{0}=\right.$ $i] \leq E_{f}\left[T / X_{0}=i\right]$. Further, from (A) it is clear that $E_{f}\left[T / X_{0}=i\right] \leq E_{f}\left[T / X_{0}=0\right]$, $\forall i$. Now under $f$ with $X_{0}=0$, we have: $T=1$ w.p. $1-p_{1}$, and for $j>1, T=j$ w.p. $p_{1} p_{2} \ldots p_{j-1}\left(1-p_{j}\right)$. Now by $\mathrm{A}(3)$,

$$
\begin{aligned}
E_{f}\left[T / X_{0}=0\right] & =\sum_{i=1}^{\infty} i \cdot p_{1} \cdots p_{i-1}\left(1-p_{i}\right) \\
& \leq \sum_{i=1}^{\infty} i \cdot p_{1}^{i-1}\left(1-p_{i}\right) \\
& \leq \sum_{i=1}^{\infty} i \cdot p_{1}^{i-1} \\
& =\frac{1}{\left(1-p_{1}\right)^{2}}<\infty
\end{aligned}
$$

where, in the first equation above, for $i=1$, the summand is to be interpreted as $\left(1-p_{1}\right)$.

Hence $E_{f_{\alpha}}\left[T / X_{0}=i\right]<\infty$, for all $i$ and $\alpha$. So, by theorem V.2.4 of Ross (1983), $V_{\alpha}(i)-V_{\alpha}(0)$ is uniformly bounded in $i$ and $\alpha$, as a result of which by theorem V.2.2 of Ross (1983), there exists a bounded function $h(i)$, and a constant $g$, such that

$$
\begin{gathered}
g+h(i)=\max \{R(i, 1)+p(i, i+1,1) h(i+1) \\
+p(i, 0,1) h(0), h(0)\},
\end{gathered}
$$

where $g=\lim _{\alpha \rightarrow 1}(1-\alpha) V_{\alpha}(0)$. Now, as a result of (7) and theorem V.2.1 of Ross (1983), there exists a stationary policy $\pi^{*}$ such that $\phi_{\pi^{*}}(i)=\max _{\pi} \phi_{\pi}(i)=g, \forall i \geq 0$, and is the one which for each $i$, prescribes an action that maximizes the right side of (7). Again by theorem V.2.2 of Ross (1983), $\exists\left\{\alpha_{n}\right\}$ of discount factors such that $\alpha_{n} \in(0,1), \forall n$ and $\alpha_{n} \uparrow 1$ as $n \rightarrow \infty$ and $h_{\alpha_{n}}(i) \rightarrow h(i)$ which is a bounded function, and where $h_{\alpha_{n}}(i)=V_{\alpha_{n}}(i)-V_{\alpha_{n}}(0)$. By proposition $1, h_{\alpha_{n}}(i) \leq 0, \forall n, i$. Hence, $h(i) \leq 0 \forall i$. Also, $h_{\alpha_{n}}(0)=0, \forall n$, and hence $h(0)=0$. As a result, (7) becomes

$$
g+h(i)=\max \left\{R(i, 1)+p_{i+1} \cdot h(i+1), 0\right\} .
$$

So, the optimal policy is one which switches to control 0 , at the first instant at which

$$
R(i, 1)+p_{i+1} h(i+1) \leq 0,
$$


or

$$
(A+B) p_{i+1}-B+p_{i+1} h(i+1) \leq 0
$$

or

$$
p_{i+1} \leq \frac{B}{A+B+h(i+1)}
$$

Let

$$
\hat{i}=\min \{i \mid(9) \text { holds }\} .
$$

If the set in (10) is empty, we take $\hat{i}=\infty$.

The above is summarized in the following theorem, which is the main result of this section.

Theorem 1. Under $(A)$, there exists a state $\hat{i} \leq \infty$ given by (10), such that the long run average reward optimal policy selects control 0 when the Markov chain is in $\hat{i}$.

\section{Discussion of the results}

(1) The function $h(i)$ is monotonically nonincreasing in $i$. This is because, by proposition $1, V_{\alpha_{n}}(i+1) \leq V_{\alpha_{n}}(i) ; \forall i$, and where $\left\{\alpha_{n}\right\}$ is the subsequence of discount factors $\alpha \in(0,1)$ defined earlier such that $h_{\alpha_{n}}(i) \rightarrow h(i) \forall i$. Then, $V_{\alpha_{n}}(0)-V_{\alpha_{n}}(i+1) \geq$ $V_{\alpha_{n}}(0)-V_{\alpha_{n}}(i)$. Taking limits on both sides as $n \rightarrow \infty$, we obtain $h(i+1) \leq h(i)$ $\forall i$.

(2) As a result of above, note that the RHS of (9) increases with $i$, whereas the LHS of (9) decreases with $i$ and so, we would only be interested in the first $i$ at which the RHS overshoots the LHS.

(3) $R(0,1)>0$ corresponds to $p_{1}>B /(A+B)$. For this case, consider a policy $f$ (say) which when starting in state $i$ selects action 1 if $R(i, 1)>0$, till the first $k$ such that $R(i+k, 1) \leq 0$ (which one expects by lemma 1) and selects action 0 after that, with the same thing repeated from state 0 subsequently. If $V_{\alpha}^{f}(i)$ is the cumulative $\alpha$-discounted reward function corresponding to this policy when starting in state $i$, then clearly $V_{\alpha}^{f}(i) \geq 0$ and so $V_{\alpha}(i) \geq V_{\alpha}^{f}(i) \geq 0$. Further if $R(0,1) \leq 0$, then $R(i, 1) \leq 0, \forall i$, and hence it would be optimal to have a policy which selects action 0 for ever, starting in any state. It can be formally argued in this case that $V_{\alpha}(i)=0, \forall i$. Hence, we conclude here that irrespective of $R(0,1)$ being positive or negative, $V_{\alpha}(i)$ is always nonnegative for every $i$.

(4) From (9), define $\left\{\bar{p}_{\alpha_{n}}(i)\right\}$ as:

$$
\bar{p}_{\alpha_{n}}(i)=B /\left(A+B+V_{\alpha_{n}}(i)-V_{\alpha_{n}}(0)\right)
$$

where $\left\{\alpha_{n}\right\}$ is defined as before. To check that it is a probability, we need to check that $0 \leq \bar{p}_{\alpha_{n}}(i) \leq 1, \forall i$. Now for $\bar{p}_{\alpha_{n}}(i) \leq 1$, we need to check if $A+V_{\alpha_{n}}(i)-V_{\alpha_{n}}(0) \geq 0$. From (3), note that $V_{\alpha_{n}}(i) \geq \alpha_{n} . V_{\alpha_{n}}(0)$. Further as in point (3) above, $V_{\alpha_{n}}(0) \geq 0$, $\forall \alpha_{n}$. Hence, 


$$
A+V_{\alpha_{n}}(i)-V_{\alpha_{n}}(0) \geq A-\left(1-\alpha_{n}\right) V_{\alpha_{n}}(0)
$$

Now, as in $\S 3, V_{\alpha_{n}}(0) \leq|R(0,1)| /\left(1-\alpha_{n}\right)$. If $R(0,1)<0$, then $V_{\alpha_{n}}(0)=0$ and we are done. So, let $R(0,1) \geq 0$. Then, since $-V_{\alpha_{n}}(0) \geq-R(0,1) /\left(1-\alpha_{n}\right)$, we have from (11),

$$
\begin{aligned}
A+V_{\alpha_{n}}(i)-V_{\alpha_{n}}(0) & \geq A-R(0,1) \\
& =A-(A+B) p_{1}+B \\
& =A\left(1-p_{1}\right)+B\left(1-p_{1}\right)>0 .
\end{aligned}
$$

Further, for $\bar{p}_{\alpha_{n}}(i) \geq 0$, we need denominator to be positive, and this follows immediately from above. Now, $\bar{p}_{\alpha_{n}}(i) \rightarrow \bar{p}(i), \forall i$, as $\alpha_{n} \uparrow 1$, where $\bar{p}(i)$ is the RHS of (9). Hence, $0 \leq \bar{p}(i) \leq 1, \forall i$. It is hence, a probability.

(5) Let us consider the cases $B=0$ and $A=0$ separately. $B=0$ corresponds to ignoring fuel loss which is reflected from our optimal policy, since then, $\bar{p}(\hat{i})=0$ and so, it is optimal to keep sending fuel for ever. This is what one expects from intuition, for maximizing power. The case $A=0$ corresponds to ignoring power output of the engine. Note that $\bar{p}_{\alpha_{n}}(i)=B /\left(B+V_{\alpha_{n}}(i)-V_{\alpha_{n}}(0)\right)$, in this case. However, $R(0,1)=B p_{1}-B=B\left(p_{1}-1\right)<0$. Hence, $V_{\alpha_{n}}(i)=V_{\alpha_{n}}(0)=0$, as mentioned in point (3) above. So, $\bar{p}_{\alpha_{n}}(i)=1, \forall i, n$, and hence $\bar{p}(i)=1, \forall i$, again as expected, since it tells us that 'never to send fuel' policy is optimal.

(6) Finally, we look at the problem of explicitly computing $g$ and $h(i)$. By assumption $\mathrm{A}(3)$, we have $0<1-p_{1} \leq 1-p_{2} \leq \ldots$. Consider a new process with identical state and action spaces and identical rewards, but with transition probabilities given by,

$$
p(i, i+1,1)=\frac{p_{i+1}}{p_{1}}, \quad p(i, 0,1)=1-\frac{p_{i+1}}{p_{1}}=\frac{p_{1}-p_{i+1}}{p_{1}}
$$

and $p(i, 0,0)=1, \forall i \geq 0$. Let $\bar{V}(i)$ be the $p_{1}$-discount optimal value function for this new process, where $p_{1}$ is treated as a discount factor. The analysis of (Ross 1983), pp. 98-99, carries through now, and we obtain

$$
g=\left(1-p_{1}\right) \bar{V}(0), \quad h(i)=\bar{V}(i)-\bar{V}(0)
$$

The long-run average return optimal policy is one which selects action 0 , the first time $i$ such that $p_{i+1} \leq B /(A+B+(\bar{V}(i+1)-\bar{V}(0)))$. This completes our discussion of the results.

\section{Concluding remarks}

(1) We considered the problem of controlling the supply of fuel in a fuel injection internal combustion engine system, with the twin purposes of maximizing power output and minimizing fuel wastage under very general assumptions. 
(2) We formulated the problem as an infinite state controlled Markov chain on the state space of nonnegative integers using binary control variables, and applied dynamic programming techniques to obtain the long-run average reward optimal policy. The optimal policy turned out to be a threshold policy which cuts supply of fuel at a certain state $\hat{i}$ mentioned in theorem 1 . As a result if $\hat{i}<\infty$, the overall Markov chain under this policy becomes finite state with state space $\{0,1,2, \ldots, \hat{i}\}$.

(3) We fully analysed the optimal policy. Our analysis showed that the optimal policy actually gives the threshold probability. We also considered cases in which we reduce the problem either to one of maximizing power output alone or to one in which just fuel wastage is minimized, and found that in either case the threshold policy is what one expects from intuition.

(4) Finally, we looked at the problem of explicitly computing the long-run average reward and the threshold probability. We obtained expressions for both of them in terms of $p_{1}$-discount optimal value function of a modified process, which can be easily computed. Further investigation could be carried out for the experimental verification of the algorithm.

SB is indebted to Prof Vivek S Borkar for suggesting the approach to the problem and for many helpful discussions during the course of this work.

\section{References}

Jones V K, Ault B A, Franklin G F 1995 Identification and air-fuel ratio control of a spark ignition engine. IEEE Trans. Control. Syst. Tech. 3: 14-21

Moskwa J J, Hedrics J K 1992 Modeling and validation of automotive engines for control algorithm development. ASME J. Dynamic Syst. Meas. Control 114: 278-285

Ross S M 1983 Introduction to stochastic dynamic programming (New York: Academic Press) 